\title{
Evaluation Criteria for Online Specialized indexes
}

\author{
Abdolreza Norouzi Chakoli \\ Faculty member of Shahed University, Iran \\ Noushin Ilbeigi (Corresponding Author) \\ MA in scientometrics, Policy and Planning Office, Ministry of Science, Research and Technology, Iran \\ Ameneh Rahjoo \\ MA in Information and Knowledge Science
}

Doi:10.5901/mjss.2015.v6n6s2p526

\section{Abstract}

The objective of this study, which is based on documentary method, was to provide a list of evaluation criteria for online specialized indexes. For this purpose, the printed and electronic texts having evaluated indexes were studied and criteria which are scattered in various texts on the evaluation of indexes were identified and collected. In addition, some of the criteria were directly identified through reference and identification of indexes. Thus, all of them were classified in eight classes based on the dimensions and capabilities of the indexes that were considered by criteria, and finally, a list of evaluation criteria for online specialized indexes was presented.

Keywords: specialized index, evaluation, evaluation criteria

\section{Introduction}

For many years, indexes have been used to help users for quick locating of required information. In this regard, the basic concept of the index refers to the index in the back of the book that was presented to clarify the content of the book. However, with scientific information production growth, it was necessary to propose the indexes related to the specific topics that cover a set of specialized journals or other sources and contribute to their clarification as a necessity. Subsequently, indexes came into existence in areas such as chemistry, physics, engineering, art, architecture and so on in order to help users to access a variety of resources related to the favorite topic (Norouzi Chakeli and Hassanzadeh, 2008: 43).

On the other hand, index as one of the most important data analysis tools to analyze the scientific documents has also major contribution. Hence, the creation of this tool and controlling its permanent quality is necessary for every information system.

Awareness of the existence of scientific documents in various subject areas and their information content is regarded an important factor of research development and this is possible by using indexes (Ashrafei Rizi and Kazempour, 2007:151).

In addition, the function of indexes in providing information about ongoing research, awareness of the boundaries of knowledge to conduct new researches and using background of study in new researches should not be ignored. On the other hand, indexes can be used to explain the relationship between science and the scientific map drawing. Magazines and journals as the most important sources of information providing the latest achievements in various fields of human knowledge always use indexing to organize their information content. Given the importance of indexes, identification of criteria and indicators through which specialized indexes can be assessed has great importance. Regarding the importance of evaluating the specialized indexes, this paper tried to address the concept of the index and study the texts related to the field of indexes' evaluation and then provide the inventory relevant to the evaluation criteria of specialized indexes. 


\section{The Concept of Index}

Index is the equivalent word in English for Nomayeh which is taken from the Latin word of Indic means "Predicate". Persian equivalent of index is "Fehrest" means list that is emerged from the Pahlavi word "Pehrest" and fehrest is its Arabic (Salehi and Rahimi, 2006:146) and also index is derived from infinitive which means guidance and indicating (Niyazi, 2002).

According to Bonura (1994), index is a design that indexer creates to represent the knowledge and purpose of author's text. Index is a tool for better access to information and analytical and systematic subject that is alphabetically arranged according to subject. In fact, index re-organizes the existing information in the document by extracting what is in it. Data and Sinha (1984) believe that index is the most important means in information retrieval system and also is compact guidance for information existing in the document, book or collection. In other words, index is the communicative bridge between data source content and user. Similarly, Gingrande (2004) knows index as a guide to the document, or some of the existing documents in the database that is made to reveal the nature of them.

Herri (1983) defines citation index as regular inventory of cited articles that each of them brings a list of cited articles. The citing text article is called source and cited article is named reference or document (Citation) (Osare and Farsi, 2002: 228). In general, the following characteristics can be pointed out in index definition (Ashrafi Rizi and Kazempour, 2007:153):

- Guiding names, places, concepts and items in a document or a set of documents

- Arranging available entries based on the special order (often alphabetically)

- Demonstrating the location of concepts and items having references

Based on the definitions provided, it can be concluded that the index is a tool for systematic guidance of user to a text, content, a set of documents or any recorded information that is arranged in a special form (as usual in alphabetical order). In addition, it shows the position and location of each content incurred in retrieving information by using a specific method and referential system. The order of preparation of index is to organize information for quick and easy retrieval.

\section{The Framework of Evaluation Criteria of Specialized Indexes from the Perspective of Experts}

During the last hundred years, most of thematic indices were alphabetically regulated merely as a list of words, without or with subdivision along with a simple hierarchical structure, containing two or three levels. During this period, although many scholars and experts were regarded index valuable in the documents and additionally, as a major need for literature search and other collections, any mass movement to ensure the provision of high-quality indices was not performed. Along with these requirements, the index quality is rarely discussed by publishers or users and most of them request non-specialists and unskilled administrative staff to do text indexing. Thus, for many years, there is no specific and coherent criteria to evaluate the advantage of index, only in recent years, information sciences scholars compiled such criteria (Niyazi, 2002:18-42).

Currently, most experts believe that the index should be complete and specific enough and makes aware the user from all aspects of a particular topic. The point that should be considered before the index evaluation is to create criteria based on the needs of users. The aim of index is to contribute the user to find information due to the time and cost constraints and personal effort. Therefore, information should be retrieved as much as possible in order to be relevant to users' needs. Accordingly, if an index will not lead to suitable information retrieval, the information system will fail. Therefore, the index evaluation at all levels is a necessity. Among the important factors involved in making a good index that should also be considered in determining its criteria assessment can be pointed to the correctness, completeness and flexibility of entries based on dynamic language. Thus, a good index can be expected to be easily read, its contents to be explained in detail, to be effective from the perspective of user and contains multiple entries for a single topic. Further, index evaluation can be quantified objectively, but mental evaluation is needed for completion. For example, one of the factors considered in index evaluation is the length of keywords that should be proportional to the length of the text. In this regard, there is no quantitative standard criterion, but it can be considered subjectively and based on the nature of the subject, depth of discussion and user's preferences (Ashrafi Rizi and Kazempour, 2007:154).

Since the inherent objective of any index is indexing information resources with high quality, index manufacturers should be selected among the published information resources.

In this regard, Truelson states that quality is the main criterion for magazines selection in index medicus. The policy of index medicus in resources indexing refers to valuable articles indexing instead of all articles. The purpose of this index is to cover the best journals in all disciplines related to the topic. The number of journals indexed in a matter depends on the quality of each journal. The linguistic and regional coverage depends on the quality as well. Although, 
there are guidelines to determine the quality of the magazines, it should be noted that, generally, it is difficult to determine the quality of a journal. For this purpose, index medicus has a selection committee that before indexing publications evaluates and chooses them. The committee consists of staff of library, medical librarians and subject specialists. The committee initially determines specific and practical criteria to select publications, then identifies and collects the resources that are consistent with these criteria and finally, in addition to the systematic evaluation of these titles, evaluates them periodically (Truelson, 2006:333).

Cleveland and Cleveland have evaluated index in three levels (2001) including technical, semantic, and effectiveness. They believed that these levels are related to each other and in technical level index should have the appropriate language and understandable form that can be used easily, at the semantic level, words should convey meaning without ambiguity, and in the third level namely the effectiveness level index should correctly identify relevant information and to be effective in information retrieval.

Also, Azgaldov (1969) identifies the criteria for determining the index quality including adequacy, which encompasses a large number of features including coverage scope, features of words used in indexing and indexical factors such as holistic view and cohesion, generality, which basically refers to the diversity of explorations that can be done, ergonomocity, referring to the ease of use, currency, which is regarded as the currency rate for a tool, finally cost, which refers to the price of per index. He correctly points out that "if low index lead to the facility (i.e., ergonomocity and currency), even the most efficient printed indexes will face to the problem. The reverse is also true and the index that is simply and conveniently designed to use, even if its retrieval efficiency not to be so high, it will still gain popularity. "

In addition to the afore-mentioned issues, some experts like Lancaster believes that in general, indexes with abstracts have priority to the indexes with no abstract because this type of indexes provide more information to the user in this regard that how much a certain degree can be useful. The existence of abstract is very valuable especially in cases that the documents can be found difficult or they are presented in an unfamiliar language to user. However, abstracts are not always necessary. For example, the combination of evidence with major headings of subject and subdivisions which are normally provided in the following are enough to indicate the potential relevance of the document with user's information needs.

At the same time, those indexes that only depend on the words of title, provide limited facilities to retrieval should be accepted (Lancaster, 2003:263-264). On the other hand, since online specialized indexes are also considered as a kind of bibliographic databases, they should also be consistent with some evaluation criteria of those databases. In this context, Allison introduces criteria to select bibliographic databases based on their characteristics. These criteria are used as a part of the evaluation criteria of specialized indexes. Accordingly, a high quality specialized index which is a bibliographic database should have traits associated with the following dimensions (Allison, 2000: 56-63):

Output options: display, print, save, e-mail, being user-friendly and document delivery

Search interface: stability, differences between editions (Non-network, local network, the web, etc.), user-friendly interface based on user needs, integrated access/ harmonized with standard of $3950 Z$ and help pages.

Options of search: Boolean and proximity operators, searching for a particular field, thesaurus / directory, side search, limit, storage / compound search, different search methods, multiple database searches, various years search, and help system search.

Content: Access to the full-text files of past, pdf format, graphics / images and being common / currency

Local issues of library: communicating with internal set, interlibrary limitations of loan, credit (only for remote access), applied and prepare programs, and guidance and support hours.

In addition, a bibliographic database cannot be evaluated only through its ability to meet the information needs of users. According to Lancaster, a database in relation to the needs of particular information can be assessed based on the following four criteria (Lancaster, 2003: 193-194):

a. Coverage scope: how much do the published literature on a specific topic in a certain period exist in the database?

b. Retrievability: how much can the literature published in a special subject that exists in the database be retrieved by search strategies?

c. Predictability: how much a user can judge about the appropriateness or inappropriateness of document by using the existing information in the database?

d. Timeliness: can the new published resources be retrieved, or the delay in indexing/ abstracting caused the search results contain old documents?

From the viewpoint of evaluator's timelines, efficiency criterion has many attractions. Timeliness measurement is relatively simple and after measurement, it is non-controversial because it does not depend on subjective and individual judgments (Lancaster, 2003: 220). Also, Lancaster believes that the use of performance measures such as recall and 
precision are very useful in studies of retrieval from a variety of printed and electronic databases (Lancaster, 2003: 224).

Theoretically, comparison of indexes and abstracts with published standards is the best way for evaluation. In general, a standard is compiled to compare what it is with what it should be and it could be considered as a verified pattern. Standard is applied in various areas such as indexing which aims to increase the indexes quality. There are different standards in the field of indexing that are created by national or international organizations. Most of the existing standards in the field of indexing emphasize on the final outcome (index or abstract) and pay less attention to the indexing way and how it is done. Some of the most important indexing standards are presented in the following Table (Cleveland and Cleveland, 2001).

Table 1. Standards for indexing area

\begin{tabular}{|l|c|c|}
\hline Application area & Year of establishment & Standard title \\
\hline Basic standard for indexes & 1984 & ANSI /Z 39.4 \\
\hline Information retrieval standard: applications, definition, service and protocol (version 3) & 1995 & ANSI/NISO Z 39.50 \\
\hline Index preparation standard for books, serial publications and other documents & 1988 & BS 3700 \\
\hline Documents evaluation standard, determining their subjects and selection of indexical terms & 1984 & BS 6529 \\
\hline Documents analysis standard, determining subjects, and selection of indexical terms & 1985 & ISO 5963 \\
\hline Standard and instruction of content, structure and presentation of indexes & 1996 & ISO 999 \\
\hline
\end{tabular}

\section{The Final Criteria for the Specialized Indexes Evaluation}

As it was already mentioned, according to the researchers' ideas, the final criteria for the specialized indexes evaluation should be in a way that ultimately leads to the identification of high quality indexes. Since the high quality scientific documents can be identified and evaluated by using high quality indexes, the application of these criteria is a necessity. These criteria can be appropriate for both information users who are looking for the high quality information sources and for index manufacturers who are seeking to improve their products level. Furthermore, these criteria can be useful and usable for research managers of universities and research centers that always need to have criteria to identify the valid indexes in order to validate the scientific productivity of their faculty members. On the other hand, these criteria can be useful for libraries and information centers considering the indexes with collective targets and leading them to identify the high quality indexes. In this way, the main criteria that can be applied by index users, manufacturers and librarians to evaluate the indexes are as follows:

The clarity of purpose and index domain: it refers to some factors such as having clear and applied criteria to select information sources for indexing, specifying the utilized principles and standards in adjustment of index records, having the policy relevant to currency interval and its process, writing index policy in relation to the copyright laws, determining the subject scope covered by index, specifying types of documents covered by the index, defining the languages covered by index, and explaining the geographical area covered by the index.

Index manufacturers' validity: it refers to some factors such as reputation of index creators in intended subject area, reputation of index publisher and supporter in the desired subject area, the index with active editorial board consisting of critic and experienced referees with a high professional position in the desired subject area, and dependence of index to a reputable organization.

Sources covered by the index: it considers some variables including continuous and systematic evaluation of information sources to cover the index, evaluating the information sources with regard to the relevant subject group, information sources marketing covered by index periodically, completeness of scope covered by index in the desired subject area with regard to the presented objectives, the index with retrospective coverage, internationality of the language of information sources covered by index, and internationality of the geographical area of information resources covered by index.

The organization (storage) of information in index : it pays attention to some issues like indexing documents in the shortest time possible after their publication, using subject headings or important and credible thesauri in the intended subject area, the proportion of selected terms in index with subject expertise of users, the existence of referral links (outsourcer textual) in index to access to more resources, lack of blind links in index, and the existence of a variety of indexes in index (title, author, subject index, etc.)

Search capabilities in index: it evaluates some factors in index including the retrieval possibility of required options in index (integrity), the possibility of non-retrieval of unwanted options in index (precision), the possibility of conducting search in multiple databases simultaneously, the ability to perform simple and advanced search, the ability to 
search through browse, the possibility of searching in natural language, the ability to select and execute searches directly by using the thesaurus in index, the possibility of searching with the keywords of title, subject, and author, etc., the ability to use arithmetic operators in index, the possibility of taking advantage of root search operators and global search (truncation and proximity operators), the ability to formulate search, the possibility to utilize the suitable limitations such as year of publication, document type, language, etc., and the possibility to simultaneous search among the records of previous years and the current year.

Displaying information and search results in index: it considers the completeness of the retrieved bibliographic records in index, the existence of accuracy and precise in the retrieved bibliographic records, lack of spelling and grammatical errors in the retrieved bibliographic records, the possibility to provide search results in an understandable format to the user, prominence of retrieved results, searched terms or synonyms, the ability to sort search results according to specific fields such as author's name, date of publication, type of document, etc. in index, the possibility to limit search results by user in index, the ability to process and combine of search results together, the possibility to save or label search results for future, the possibility to jump to a specific record of the search results in index, definiteness of number of retrieved documents in index, the ability to print based on the different needs of users (printing the selected text, the whole document, print option), the ability to send search results by e-mail, and the possibility to view a history of searches carried out in index.

Being user-friendly index: it evaluates factors such as having a simple and user-friendly structure in index, the constant database and low changes in the user environment in index, having good design such as color, readable font, proper spacing, color contrast of background and context, using header tags and description to better understanding, using assistant tools like icons, buttons, rising menus, navigation symptoms, search training, and example to browse the index page, easily jump to the previous and next pages, the possibility of providing technical assistance and support services by e-mail, phone or proposing common questions, considering provisions to educate users (Demo, Free Trial option, etc.) in index, the existence of searchable guidance, the possibility of dissemination of selective information, timelineness of the currency in index, the possibility to access to resources and references used in the proof by the link, the possibility of sharing communication directly from citations to full-text electronic journals, the ability to access to document by document delivery services, or to order online degree if there is no access to the text of the document, providing information like email, phone, fax, and postal address to call the author or publisher of index, providing abstract in addition to the bibliographic information for the information sources, unchanged index address (URL) frequently, the existence of a link to the new address in the event of change in address, having graphical facilities, using video and audio features for better understanding of the text, providing index map on the site, meaningfulness of the messages expressed in index, last updated visibility in all pages, the existence of descriptive information about each link, quick load of hypertext links, home page, links within text, the existence of search box on the home page, the ability to browse different indexes and select records among these indexes, using within text links in index, and receiving additional subscription costs to access to textual resources that are not accessible through database.

Index flexibility: it refers to some criteria including the possibility of correcting spelling and grammatical errors and suggesting alternative words, the possibility of selecting different formats of retrieved sources such as PDF, TEXT, HTML, the ability to transfer the information to other software packages, the ability to convey search results to Ski and video files and send them, the possibility of selecting some of the fields for output search fields which is different from the default database fields, the ability to change the names of fields in the index, and the possibility of having access to index through the types of browsers and operating systems.

\section{Conclusion}

Although, due to the importance of evaluation criteria for online specialized indexes, some criteria were provided to identify these types of indexes in the present study, it should be noted that all these criteria should be regarded simultaneously in order to judge about a good index. In other words, these criteria are considered as a whole and only if, all of them to be used together, the actual and away from bias results can be provided for one or more indexes. Obviously, if a few specific criteria are selected among them and be used for indexes' evaluation, not only it cannot provide reliable results to the research community, but also it can cause distortion of the facts existing in indexes.

\section{References}

Allison, D.A., Mcneil, B., \& Swanson, S. (2000). Database selection: One size does not fit all. College \& Research Libraries, 56-63. Ashrafi Rizi, H., \& Kazempour Z. (2007). Evaluating index and indexing: Criteria and Standards. Information Science and Technology, 
23, 151-168.

Azgaldov, E.G. (1969). A framework for description and classification of printed subject indexes. Libri, 19, 275-291.

Bonura, L. S. (1994). The art of indexing. New York: John Wiley \& Sons.

Cleveland, D. B., \& Ana D. C. (2001). Introduction to indexing and abstracting. $3^{\text {rd }}$ ed. Englewood: Libraries Unlimited.

Dutta, S., \& Sinha, P.K. (1984). Pragmatic approach to subject indexing: A new concept. Journal of the American Society for Information Science. 35 (6), 325- 331.

Gingerande, A. (2004). The Value of Indexing. AlIM E-Doc Magazine, 18 (2), 9.

Lancaster, F. (2003). Indexing and abstracting: Theoretical and practical foundation. Trans: Gilvari, A., Tehran: Chapar.

Niyazi, S. (2002). Indexing. In Encyclopedia of Library and Information, Tehran: National Library, 2, 1840-1852.

Norouzi Chakeli, A.R., \& Hasanzadeh, M. (2008). Citation indexing and scientific relations. Approach, 43, 15.

Osareh, F., \& Farsi, Gh.A. (2002). Science Citation Index (SCI): Structure and its Applications. Approach, 27, 232-226.

Salehi, K., \& Rahimi, H. (2006). Explaining the process of evaluation of journals in Institute for Scientific Information (ISI). Journal of book, 66, 141-160.

Truelson, S.D. (2006). What the index medicus indexes, and why?, New Haven: Yale Medical Library. p. $329-336$. 